\begin{tabular}{|c|l|}
\hline Title & Visualization of Ras PI3K interaction in the endosome using BiFC \\
\hline Author(s) & Tsutsumi, Kaori; Fujioka, Y oichiro; Tsuda, Masumi; Kaw aguchi, Hideaki; Ohba, Y usuke \\
\hline Citation & $\begin{array}{l}\text { Cellular Signalling, 21(11), 1672-1679 } \\
\text { https://doi.org/10.1016/.cellsig.2009.07.004 }\end{array}$ \\
\hline Issue Date & 2009-11 \\
\hline Doc URL & http://hdl.handle.net/2115/54563 \\
\hline Type & article(author version) \\
\hline File Information & Cell Signal_21(11)_1672-1679.pdf \\
\hline
\end{tabular}

Instructions for use 


\section{Visualization of Ras-PI3K interaction in the endosome using BiFC}

Kaori Tsutsumi $^{\text {a,b }}$, Yoichiro Fujioka ${ }^{\text {a,1 }}$, Masumi Tsuda ${ }^{\text {a,1 }}$, Hideaki Kawaguchi ${ }^{\text {a }}$, Yusuke Ohba ${ }^{\text {a,* }}$

${ }^{a}$ Laboratory of Pathophysiology and Signal Transduction, Hokkaido University Graduate

School of Medicine, Sapporo, Japan

${ }^{\mathrm{b}}$ Department of Biomedical Science and Engineering, Faculty of Health Sciences, Hokkaido University, Sapporo, Japan

* Corresponding author. Laboratory of Pathophysiology and Signal Transduction, Hokkaido University Graduate School of Medicine, N15W7, Kita-ku, Sapporo 060-8638, Japan. Tel.: +81-11-706-5158; fax: +81-11-706-7877.

E-mail address: yohba@med.hokudai.ac.jp

${ }^{1}$ These authors contributed equally to this work

Keywords: Ras; PI3K; endosome; BiFC 


\begin{abstract}
Recent studies indicate the importance of spatiotemporal regulation in the diversity and specificity of intracellular signaling. Here, we show that Ras-PI3K signaling plays an important role in the local regulation of phosphatidylinositol metabolism in the endosome through live-cell imaging by using a bimolecular fluorescence complementation technique, in which molecular interaction is indicated by fluorescence emission. Using several possible combinations of Ras and the Ras binding domain, we identified an optimal set of probe molecules that yielded the most significant increase in fluorescence intensity between the active and inactive forms of Ras. This combination revealed that, among the Ras effectors tested, phosphatidylinositol 3-kinase (PI3K) was specifically implicated in signaling in the endosome. We also found that full length PI3K was recruited to the endosome in EGF- and Ras-dependent manners, which appears to be essential for the activation of PI3K in this compartment. Taken together, these findings demonstrate that the spatiotemporal regulation of Ras-PI3K signaling may dictate the activation of PI3K and subsequent downstream signaling in the endosome.
\end{abstract}




\section{Introduction}

The Ras proteins, consisting of $\mathrm{H}-, \mathrm{K}-$, and N-Ras, are monomeric GTPases that regulate a variety of signal transduction cascades involved in cell growth, differentiation, survival, and motility [1-3]. The GTP-bound, active form of Ras interacts with the conserved Ras-binding domain (RBD) of its effector molecules, which include c-Raf1 serine/threonine kinase, phosphatidylinositol 3-kinase (PI3K), and Ral guanine nucleotide dissociation stimulator (RalGDS). Currently, more than ten different effectors have been identified, and this variety is responsible for the many facets of Ras functions [4]. The precise mechanism by which Ras exploits different effectors, however, remains poorly understood. Spatial regulation of Ras may constitute one of the possible mechanisms through which Ras elicits heterogenous signals by binding to different effectors in distinct cellular locations [5].

Intracellular localization of Ras is determined by a membrane-targeting region at its Cterminus ('CAAX' box or farnesylation moiety), and targeting to the plasma membrane (PM) is crucial for its biological activity [6]. The variations in the amino acid sequences within the hypervariable region adjacent to the processed CAAX motif can modulate the ultimate localization in various membranes. For example, in the case of K-Ras, a poly-lysine motif serves as the secondary PM targeting signal [7]. In contrast, a palmitate moiety at the cysteine residue(s) of $\mathrm{H}$-Ras and $\mathrm{N}$-Ras serves as the targeting signal for the endomembrane compartments, including the Golgi apparatus [8]. Therefore, H-Ras and N-Ras localize to the Golgi and other vesicular structures in addition to the PM. Bivona et al. reported that the noncanonical PLC $\gamma$-CalDAG-GEFII /RasGRP1 pathway activates H-Ras at the Golgi to promote neurite outgrowth of PC12 rat pheochromocytoma cells [9]. More recently, H-Ras was shown to localize in the endosomes $[10,11]$, but the functional significance of this localization remains obscure. Importantly, all of these observations are due to the recent development in imaging technologies. 
The discovery of the green fluorescent protein (GFP) and its subsequent engineering into useful variants have facilitated direct visualization of protein complexes in living cells, thus enabling the investigation of protein interactions in their physiological environment (reviewed in [12]). Two principal methods have emerged to visualize interactions of protein complexes in living cells. The first technique, known as fluorescence resonance energy transfer (FRET), is based on the radiationless transfer of excitation energy between two fluorophores that are brought sufficiently close together [13-15]. The second technique, known as bimolecular fluorescence complementation (BiFC), is based on the formation of a fluorescence complex generated by the reconstitution of two fragments of a fluorescent protein, via a direct association of two target proteins that are each tagged with half of the aforementioned fluorescent protein. Hence, reconstitution of the fluorescent protein is enabled by the direct interaction between the two target proteins $[16,17]$. One of the advantages of $\mathrm{BiFC}$ is that a specific interaction produces a single color, making it amenable to combination with other fluorescent proteins and imaging techniques.

Here, using BiFC, complemented with immunofluorescence and other fluorescent proteins, we found that Ras-PI3K signaling happens specifically in the endosome, and appears to be required for the accumulation of phosphatidylinositol 3,4,5-triphosphate $\left(\mathrm{PIP}_{3}\right)$ in the endosome. 
Tsutsumi, K. et al.

\section{Materials and Methods}

\subsection{Expression plasmids}

The cDNA for Venus, a variant of the yellow emitting mutant (YFP) of the enhanced green fluorescent protein (GFP) (obtained form A. Miyawaki, RIKEN, Wako, Japan), was amplified by PCR using a VNn forward primer, 5'-

CCGAATTCGCCATGGTGAGCAAGGGCGAG -3', and a VNn reverse primer, 5'GGCTCGAGGATGTTGTGGCGGATCTTGA -3', in addition to a VCn forward primer, 5'CCGAATTCGCCATGGAGGACGGCGGCGTG -3', and a VCn reverse primer, 5'GGCTCGAGGTACAGCTCGTCCATGCCGA -3' to produce cDNA corresponding to the Nterminal 1-172 amino acid residues and the C- terminal 173-238 amino acid residues of Venus, respectively (referred to as $\mathrm{VN}$ and $\mathrm{VC}$, hereafter). The resulting PCR products were digested with EcoRI and XhoI and inserted into the EcoRI-XhoI site of pCAGGS-EGFP-H-Ras (wild type and G12V) [18] to generate pCAGGS-VN-H-Ras and pCAGGS-VC-H-Ras. The Ras-binding domains (RBDs) of human c-Raf1 (obtained from S. Hattori, Kitasato Univ., Japan), human PI3K p110 (form L. Stephen, UK), and RalGDS (amplified from a human endothelial cDNA library) were subcloned into the XhoI-NotI site of pCAGGS-VN-H-Ras, pCAGGS-VC-H-Ras, and pCXN2-Flag-H-Ras [18] to generate pCAGGS-VN-RBDs, pCAGGS-VC-RBDs, and pCXN2-Flag-RBDs, respectively. VN and VC were also amplified using the following primers: a VNc forward, 5'- CCGCGGCCGCATGGTGAGCAAGGGCGAGG -3', and a VNc reverse, 5'- GGAGATCTTCAGATGTTGTGGCGGATCTTG -3'; a VCc forward, 5'CCGCGGCCGCGAGGACGGCGGCGTGCAGCT-3', and a VCc reverse, 5'GGAGATCTTCACTACAGCTCGTCCATGCCG -3', respectively, and subcloned into the NotIBglII site of pCXN2-Flag-RBDs to generate pCXN2-Flag-RBDs-VN and pCXN2-Flag-RBDsVC. pHcRed1-C1 was purchased from Clontech (Mountain View, CA, USA) and used in some experiments as a control to normalize the expression level. To obtain HA-tagged PI3K p110 the coding sequence of $\mathrm{p} 110 \gamma$ was amplified by PCR, digested by XhoI and NotI, and subcloned 
into a pCAGGS-3HA vector [19]. cDNA of human Akt was a kind gift from Y. Gotoh (Univ. Tokyo, Tokyo, Japan). The plextrin homology (PH) domain was amplified as reported previously [20], and subcloned into the XhoI and NotI site of the pCAGGS-ECFPC vector [21]. Expression vectors for H-Ras S17N, G12V E37G, G12V D38E, and G12V Y40C were kindly provided by M. Matsuda (Kyoto University, Kyoto Japan).

\subsection{Reagents and antibodies}

Recombinant human epidermal growth factor was purchased from PeproTech (Rocky Hill, NJ, USA). AlexaFluor 488- and AlexaFluor 594-conjugated goat anti-mouse antibodies, and AlexaFluor 647-conjugated chicken anti-rat antibody were obtained from Invitrogen (Carlsbad, CA, USA). Anti-Myc, anti-HA (3F10), and anti-EEA1 monoclonal antibodies were obtained from MBL (Nagoya, Japan), Roche (Indianapolis, IN, USA), and BD Biosciences (San Jose, CA, USA) respectively.

\subsection{Cell culture and transfection}

293T and Cos-1 cells were maintained in Dulbecco's modified Eagle's medium (DMEM, Sigma) supplemented with $10 \%$ fetal bovine serum (FBS, Cansera, Canada) at $37{ }^{\circ} \mathrm{C}$ in a humidified atmosphere containing $5 \% \mathrm{CO}_{2}$. Expression plasmids were introduced into the cells using FuGene HD (Roche) according to the manufacturer's protocol. Of note, to avoid the dominant negative effect of forced expression of RBDs, the amounts of plasmids for RBDs are limited to one-tenth of those for Ras.

\subsection{Fluorescence microscopy}

Cells were imaged with an IX71 inverted microscope (Olympus, Tokyo, Japan) equipped with BioPoint MAC5000 excitation and emission filter wheels (Ludl Electronic Products Ltd., Hawthorne, NY, USA), and a Cool SNAP-HQ cooled CCD camera (Roper Scientific, Trenton, NJ, USA) as described previously [22]. Excitation and emission filters used in this study include 
Tsutsumi, K. et al.

XF1068 (500AF25) and XF3079 (535AF26) (Omega Optical Inc. Brattleboro, VT) for YFP, XF1071 (440AF21) and XF3075 (480AF30) (Omega) for CFP, and BP520-550 and BA580IF (Olympus) for HcRed. An XF2034 (455DRLP) dichroic mirror was used throughout the experiments. Cells were illuminated with a 75-W Xenon lamp through a 6\% ND filter. MetaMorph software (Molecular Devices, West Chester, PA, USA) was used for control of the CCD camera and filter wheels and also for the analysis of the cell image data.

Cells plated on a collagen-coated 35-mm-diameter glass-base dish (Asahi Techno Glass Co., Tokyo, Japan) were transfected with expression vectors. After 24 h, cells were serumstarved in phenol red-free DMEM/F12 (Invitrogen) for $4 \mathrm{~h}$ and placed in a chamber box on a microscope, in which the temperature was maintained at $37^{\circ} \mathrm{C}$. Fluorescence images were recorded at 30 second intervals. Starting at $20 \mathrm{~min}$, cells were stimulated with $100 \mathrm{ng} / \mathrm{ml} \mathrm{EGF}$. In some experiments, cells were pretreated by incubation at $16{ }^{\circ} \mathrm{C}$ for $1 \mathrm{~h}$ before EGF stimulation. Immunofluorescence microscopy was performed as described previously [18], with the exception that an FV-1000 confocal microscope (Olympus) was used in the experiments reported herein.

\subsection{Statistical analyses}

All data represent means and standard deviations and were subjected to one-way analysis of variance, followed by comparison by Student's t-tests. $P$ values obtained from the tests are reported in the figure legends. 


\section{Results}

\subsection{Development of a BiFC system for Ras-effector binding in living cells}

We first developed a series of expression vectors for the BiFC system, to detect Ras binding to its effector molecules in living cells. N- and C-terminal Venus constructs, corresponding to amino acid residues 1-172 and 173-238, respectively (VN and VC), were fused to Ras or the Ras-binding domain (RBD) of c-Raf1 (Raf-RBD) (Fig. 1a). Since the C-terminus of Ras includes a CAAX motif, which is known to be important for its intracellular localization [6], Ras was tagged only at its N-terminus. The fluorescence intensity in the cells expressing H-Ras G12V, a constitutively active form that preferentially binds to its effectors, is expected to be higher than that of wild-type-expressing cells. Among the four possible combinations, only VNRas with VC-RBD and VN-Ras with RBD-VC demonstrated an increase in fluorescence intensity in a manner dependent on Ras activity (Fig. 1b). The combination of VN-Ras and RBD-VC achieved the most significant difference between H-Ras G12V and H-Ras WT, and it was therefore utilized in the following experiments.

\subsection{Subcellular localization of the BiFC signal from Ras and its effectors}

To extend the BiFC imaging to other effector molecules, we also constructed expression vectors for RBDs of PI3K p110 (simply referred to as PI3K, hereafter) and RalGDS, and observed their intracellular distribution by confocal microscopy. As shown in Fig. 2a, the fluorescence signals were detected mainly at the PM, the established platform for Ras to be localized and activated [6]. Perinuclear distributions, in addition to those at the PM, of the fluorescence were also observed (data not shown), which we think is likely to be the Golgi apparatus, another known compartment for Ras activation [8, 9, 23]. In the cells expressing $\mathrm{H}-$ RasG12V and PI3K-RBD, vesicular structures were preferentially detected in their cytoplasm (Fig. 2b, left panels). We thus performed the BiFC assay in combination with immunofluorescence microscopy, and found that the BiFC signal generated by Ras and PI3K- 
RBD was merged with punctate staining of early endosomal antigen-1 (EEA-1), an early endosomal marker (Fig. 2b), whereas the signal from H-Ras G12V-Raf-RBD or H-Ras G12VRalGDS-RBD colocalized with EEA-1 less efficiently (Fig. 2b; see also Fig. 2c for quantitative data). These results indicate that, in the presence of RBD of PI3K, but not that of Raf or RalGDS, Ras is recruited in the endosome.

\subsection{Ras-PI3K BiFC fluorescence is associated with endocytotic trafficking}

The BiFC signal from H-Ras WT and PI3K-RBD also displayed vesicular structures that are stained by EEA-1 in cells stimulated by EGF (Fig. 3a). Quantitative analysis revealed that such vesicular structures were preferentially observed in cells expressing PI3K-RBD, but not Raf- or RalGDS-RBD as assessed using constitutively active H-Ras, and that EGF stimulation highly upregulated this colocalization (Fig. 3b). These observations raise the possibility that Ras binds to PI3K in the endosome, however, certain drawbacks associated with BiFC, namely its irreversibility and the existence of a time lag between the actual protein binding and the onset of fluorescence emission [12], prevent us to ascertaining whether the detected BiFC signal at a particular site duly reflects the molecular interaction there or not; the possibility that a complex already formed in other places is translocated and emanates fluorescence in a different location exists. To resolve this issue, we performed time-lapse microscopy, and found that the vesicular structures depicted by the BiFC fluorescence were uncertain before EGF stimulation, but induced by the stimulation. The vesicles became visible approximately $10 \mathrm{~min}$ after the stimulation and more intense after 30 to $40 \mathrm{~min}$ (Fig. 3c and Supplementary Movie S1), and moved from the periphery to the central regions of the cells, as displayed by kymographic analysis (Fig. 3d), confirming that Ras, bound to PI3K at the PM, is internalized and recruited to the endocytotic trafficking in response to EGF stimulation.

Below 17oC, endocytosis and other forms of vesicular transport are blocked [24]. We therefore analyzed vesicular formation upon the inhibition of endocytosis by low temperature 
incubation $\left(16^{\circ} \mathrm{C}\right)$. As shown in Fig. 3e and f, EGF failed to induce the formation of vesicular structures by Ras and PI3K-RBD in cells maintained at $16^{\circ} \mathrm{C}$ for $1 \mathrm{~h}$ prior to stimulation. These results together indicate that Ras bound to PI3K at the PM is internalized through endocytosis.

\subsection{Full length PI3K is recruited to the endosome in a manner dependent on Ras activation}

We then proceeded to examine the localization of full length PI3K by immunofluorescence. PI3K was diffusely localized in the cytoplasm of cells in the quiescent state (Fig. 4a, upper panels). Upon EGF stimulation, PI3K translocated to the endosomal compartment only in the presence of Ras (Fig. 4a, left lower panels), and not in its absence (data not shown). Similar structures were more obvious in cells expressing the constitutively active mutant of Ras (Fig. 4a, right lower panels). Furthermore, full length PI3K also colocalized with EEA-1 and expressed GFP-H-Ras G12V (Fig. 4b and c), indicating that PI3K is recruited to the endosome in a Ras-dependent manner.

\subsection{Requirement for Ras activation in the accumulation of $P_{I} P_{3}$ in the endosome}

We further investigated whether PI3K recruited by Ras is activated in the endosome or not through visualization of the PI3K product, $\mathrm{PIP}_{3}$. For this purpose, we utilized the CFP-tagged PH domain of Akt (PH-CFP), which recognizes $\mathrm{PI}(3,4,5) \mathrm{P}_{3}$ [and also $\mathrm{PI}(3,4) \mathrm{P}_{2}$ ], and hence is widely used to visualize $\mathrm{PIP}_{3}$ in living cells [25]. Accumulation of PH-CFP in vesicular structures became apparent after EGF simulation (Fig. 5a, arrows), and colocalized with EEA-1 (Fig. 5c). The expression of the dominant-negative mutant of Ras (Ras S17N) however, also abolished EGF-dependent $\mathrm{PIP}_{3}$ production in the endosome, although it did not repress its production at the PM (Fig. 5a, arrow heads; see also Fig. 5b for quantitative data). The distribution of PH-CFP in cells expressing Ras S17N failed to colocalize with EEA-1 (Fig. 5c). Quantitative analysis revealed that the expression of Ras S17N reduced the formation of PHCFP-recruited endosomes by EGF, but not EEA1 staining of vesicles where PH-CFP was absent (Fig. 5d), indicating an important role for Ras-PI3K signaling in both EGF-dependent 
Tsutsumi, K. et al.

endocytosis and subsequent $\mathrm{PIP}_{3}$ production in this compartment. Furthermore, we succeeded in the simultaneous visualization of co-localization between endosomes (EEA-1, red), BiFC (YFP), and $\mathrm{PIP}_{3}$ production (PH-CFP) by triple-color imaging in the cells stimulated by EGF (Fig. 5e and f).

Finally, we evaluated the effect of expressing of H-Ras G12V, harboring an additional mutation in its effector domain, on the accumulation of PH-CFP in the endosome. Y40C, D38E, and E37G mutants specifically bind to PI3K, Raf, and RalGDS, respectively [26-28]. Among these mutants, Y40C possessed the ability to augment the recruitment of PH-CFP to the vesicular structures (Fig. 5g). In contrast, the other mutants induced the recruitment less efficiently than RasG12V without the effector domain mutation, thus validating the functional significance of the interaction between of Ras and PI3K in the endosome. Overall, these data indicate that PI3K activation appears to be regulated in a subcellular localization-specific manner, that is Rasdependent in vesicular structures and Ras-independent at the PM. 


\section{Discussion}

In the present study, we provide evidence that Ras recruits PI3K in the endosome for the local regulation of phosphatidylinositol metabolism. Several studies have demonstrated that PI3K is required for endocytosis using the PI3K inhibitor wortmannin (reviewed in $[29,30]$ ). However, since wortmannin is capable of activating Rab5 [31], which belongs to another class of proteins that regulate endocytosis, the effect of this reagent on the involvement of PI3K in the suppression of endocytosis still remains controversial. Our results clearly depict the transportation of Ras along with PI3K-RBD, within the endosomes (Fig. 3). Also the activation of Ras was required for $\mathrm{PIP}_{3}$ production in this subcellular compartment (Fig. 5). Therefore, the phenomena observed here make a positive feedback loop between endocytosis and PI3K activation a functional reality.

The process of endocytosis is vital in a variety of pathophysiological phenomena, including substance uptake, receptor down regulation, microbial infection, and phagocytosis of apoptotic cells [32-34]. Diverse molecules have been implicated in the machinery related to endocytosis, and disruption of this machinery results in various disorders, including the loss of epithelial polarity and barrier functions $[35,36]$. The lipid content of the membranes comprising such vesicular structures is substantially different from that of other membranes in terms of their relative abundance of phosphatidylinositols. For example, switching from $\mathrm{PIP}_{2}$ to $\mathrm{PIP}_{3}$ is a crucial phenomenon during the maturation of macropinocytosis [11]. On the other hand, a recent report showed that loss of $\mathrm{PIP}_{3}$ paradoxically proceeds the acquisition of phosphatidylinositol 3phosphate in some vesicular compartments [37]. We thus postulate that the Ras-PI3K signaling pathway specifically participates in the maturation of these internal vesicles, where the Ras and PI3K complex resides. Also, our results may offer the way to finely tune the highly sophisticated endocytotic dynamics by manipulating the Ras-PI3K interaction. 
Tsutsumi, K. et al.

Since Ras proteins do not possess any enzymatic activity except for their intrinsic GTPase activity, binding to their effectors is essential for Ras to exert its biological activity. The most well-established mode of effector activation is the recruitment of Raf to the PM, which results in the activation of the extracellular signal-regulated kinase/mitogen-activated protein kinase cascade (reviewed in [38]). Although a similar mechanism might also be activating other effector molecules; in the case of PI3K, once it is recruited to the PM by Ras, it is trafficked into the endocytotic pathway, and activated to accomplish its mission in the endosome.

It has been the subject of debate whether Ras participates in the receptor tyrosine kinasemediated PI3K activation because the $\mathrm{p} 85$ regulatory subunit of class IA members possesses the SH2 domains that are able to directly bind to the YXXM motif containing a phosphorylated tyrosine residue [39]. In fact, overexpression of the dominant-negative Ras mutant partly inhibits induction of $\mathrm{PIP}_{3}$ by both EGF and nerve growth factor in cultured $\mathrm{PC} 12$ rat pheochromocytoma cells [40], and by fibroblast growth factor in neuroectoderm-derived SKF5 cells [41].

Consistently, $\mathrm{PIP}_{3}$ production at the PM is not disrupted by the expression of Ras S17N (Fig. 5a). On the other hand, a recent studies using knock-in mice where the RBD of PI3K is substituted with a mutant RBD that is unable to bind Ras has shown that Ras-PI3K binding is essential for a variety of pathophysiological events, including the development of the lymphatic vasculature [42]. In endothelial cell lineages, the Ras-PI3K pathway and the internalization of vascular endothelial growth factor (VEGF) receptor 1 are involved in VEGF-dependent endothelial cell motility [43] and angiogenesis [44]. It is thus possible that a defect in the endocytosis of receptors such as VEGF receptor 3 in lymphatic endothelial cells is the cause of the impaired lymphatic development in PI3K $\alpha$ mutant mice [42], considering the crucial role for Ras-PI3K signaling in local $\mathrm{PIP}_{3}$ production in the endosome, as shown by this study (Fig. $5 \mathrm{a}-\mathrm{d}$ and g). Since the Ras-PI3K interaction also plays a critical role in Ras-driven tumorigenesis, and since adult mice deficient in Ras binding of PI3Ks are healthy [42], development of anti-cancer therapeutics targeting this connection is easily envisaged. Hence, agents acting in an endosome- 
Tsutsumi, K. et al.

specific manner and having a dual-targeting nature: tumor initiation and lymphangiogenesis, will surely be very useful and well-tolerated. 


\section{Acknowledgements}

The authors thank M. Matsuda, A. Miyawaki, S. Hattori, L. Stephen, T. Sasaki, and Y. Gotoh for providing expression vectors, H. Sawa for use of the confocal laser-scanning microscope, N. Toyoda for technical assistance, S. Darmanin for critical reading of the manuscript, and members of our laboratory for helpful discussion. This work was supported in part by Grants-in-Aid from the Ministry of Education, Culture, Sports, Science, and Technology of Japan and from the Japan Society for the Promotion of Science, and by grants from the Japan Science and Technology Agency, Takeda Science Foundation, and the Akiyama Foundation. 


\section{References}

[1] Bos J.L. Biochim Biophys Acta 1333(2)(1997)M19-31.

[2] Downward J. Curr Opin Genet Dev 2(1)(1992)13-18.

[3] Campbell S.L., Khosravi-Far R., Rossman K.L., Clark G.J., Der C.J. Oncogene 17(11 Reviews)(1998)1395-1413.

[4] Vojtek A.B., Der C.J. J Biol Chem 273(32)(1998)19925-19928.

[5] Hancock J.F. Nat Rev Mol Cell Biol 4(5)(2003)373-385.

[6] Willumsen B.M., Norris K., Papageorge A.G., Hubbert N.L., Lowy D.R. Embo J $3(11)(1984) 2581-2585$.

[7] Hancock J.F., Paterson H., Marshall C.J. Cell 63(1)(1990)133-139.

[8] Choy E., Chiu V.K., Silletti J., Feoktistov M., Morimoto T., Michaelson D., Ivanov I.E., Philips M.R. Cell 98(1)(1999)69-80.

[9] Bivona T.G., Perez De Castro I., Ahearn I.M., Grana T.M., Chiu V.K., Lockyer P.J., Cullen P.J., Pellicer A., Cox A.D., Philips M.R. Nature 424(6949)(2003)694-698.

[10] Jura N., Scotto-Lavino E., Sobczyk A., Bar-Sagi D. Mol Cell 21(5)(2006)679-687.

[11] Porat-Shliom N., Kloog Y., Donaldson J.G. Mol Biol Cell 19(3)(2008)765-775.

[12] Kerppola T.K. Nat Rev Mol Cell Biol 7(6)(2006)449-456.

[13] Zhang J., Campbell R.E., Ting A.Y., Tsien R.Y. Nat Rev Mol Cell Biol 3(12)(2002)906918.

[14] Jares-Erijman E.A., Jovin T.M. Nat Biotechnol 21(11)(2003)1387-1395.

[15] Miyawaki A. Dev Cell 4(3)(2003)295-305.

[16] Hu C.D., Chinenov Y., Kerppola T.K. Mol Cell 9(4)(2002)789-798.

[17] Hu C.D., Kerppola T.K. Nat Biotechnol 21(5)(2003)539-545.

[18] Ohba Y., Mochizuki N., Matsuo K., Yamashita S., Nakaya M., Hashimoto Y., Hamaguchi M., Kurata T., Nagashima K., Matsuda M. Mol Cell Biol 20(16)(2000)60746083. 
[19] Inuzuka T., Tsuda M., Tanaka S., Kawaguchi H., Higashi Y., Ohba Y. Cancer Res 69(4)(2009)1678-1684.

[20] Nishio M., Watanabe K., Sasaki J., Taya C., Takasuga S., Iizuka R., Balla T., Yamazaki M., Watanabe H., Itoh R., Kuroda S., Horie Y., Forster I., Mak T.W., Yonekawa H., Penninger J.M., Kanaho Y., Suzuki A., Sasaki T. Nat Cell Biol 9(1)(2007)36-44.

[21] Inuzuka T., Tsuda M., Kawaguchi H., Ohba Y. Biochem Biophys Res Commun $379(2)(2009) 510-513$.

[22] Ohba Y., Kurokawa K., Matsuda M. Embo J 22(4)(2003)859-869.

[23] Chiu V.K., Bivona T., Hach A., Sajous J.B., Silletti J., Wiener H., Johnson R.L., 2nd, Cox A.D., Philips M.R. Nat Cell Biol 4(5)(2002)343-350.

[24] Punnonen E.L., Ryhanen K., Marjomaki V.S. Eur J Cell Biol 75(4)(1998)344-352.

[25] Varnai P., Bondeva T., Tamas P., Toth B., Buday L., Hunyady L., Balla T. J Cell Sci 118(Pt 20)(2005)4879-4888.

[26] Rodriguez-Viciana P., Warne P.H., Khwaja A., Marte B.M., Pappin D., Das P., Waterfield M.D., Ridley A., Downward J. Cell 89(3)(1997)457-467.

[27] Joneson T., White M.A., Wigler M.H., Bar-Sagi D. Science 271(5250)(1996)810-812.

[28] White M.A., Nicolette C., Minden A., Polverino A., Van Aelst L., Karin M., Wigler M.H. Cell 80(4)(1995)533-541.

[29] Lourdais O., Bonnet X., Doughty P. J Exp Zool 292(5)(2002)487-493.

[30] Stephens L., Ellson C., Hawkins P. Curr Opin Cell Biol 14(2)(2002)203-213.

[31] Chen X., Wang Z. EMBO Rep 2(9)(2001)842-849.

[32] De Matteis M.A., Luini A. Nat Rev Mol Cell Biol 9(4)(2008)273-284.

[33] Miller S., Krijnse-Locker J. Nat Rev Microbiol 6(5)(2008)363-374.

[34] Mizushima N., Levine B., Cuervo A.M., Klionsky D.J. Nature 451(7182)(2008)10691075.

[35] Polishchuk R., Di Pentima A., Lippincott-Schwartz J. Nat Cell Biol 6(4)(2004)297-307. 
[36] Yeaman C., Ayala M.I., Wright J.R., Bard F., Bossard C., Ang A., Maeda Y., Seufferlein T., Mellman I., Nelson W.J., Malhotra V. Nat Cell Biol 6(2)(2004)106-112.

[37] Zoncu R., Perera R.M., Balkin D.M., Pirruccello M., Toomre D., De Camilli P. Cell 136(6)(2009)1110-1121.

[38] Morrison D.K., Cutler R.E. Curr Opin Cell Biol 9(2)(1997)174-179.

[39] Rodriguez-Viciana P., Warne P.H., Vanhaesebroeck B., Waterfield M.D., Downward J. Embo J 15(10)(1996)2442-2451.

[40] Rodriguez-Viciana P., Warne P.H., Dhand R., Vanhaesebroeck B., Gout I., Fry M.J., Waterfield M.D., Downward J. Nature 370(6490)(1994)527-532.

[41] van Weering D.H., de Rooij J., Marte B., Downward J., Bos J.L., Burgering B.M. Mol Cell Biol 18(4)(1998)1802-1811.

[42] Gupta S., Ramjaun A.R., Haiko P., Wang Y., Warne P.H., Nicke B., Nye E., Stamp G., Alitalo K., Downward J. Cell 129(5)(2007)957-968.

[43] Santos S.C., Miguel C., Domingues I., Calado A., Zhu Z., Wu Y., Dias S. Exp Cell Res 313(8)(2007)1561-1574.

[44] Serban D., Leng J., Cheresh D. Circ Res 102(11)(2008)1350-1358. 


\section{Figure Legends}

Fig. 1. Development of a BiFC system to detect Ras-effector binding in living cells. (A) Schematic representation of the combination of the constructs tested to develop the detection system for the binding between Ras and the Ras-binding domain (RBD) of c-Raf1. VN and VC indicate the N-terminus and C-terminus of Venus, respectively. (B) 293T cells were transfected with a combination of expression vectors (indicated at the bottom of the graph) along with pHcRed-C1. Twenty-four hours after transfection, the cells were observed with an inverted fluorescence microscope. The YFP fluorescence intensity was normalized by the HcRed intensity with over 100 cells recorded. The average increase in fluorescence intensity of H-Ras G12V-expressing cells was compared against that of wild type (WT)-Ras expressing cells and is shown with standard deviation $(*, P<0.001)$.

Fig. 2. Distinct localization pattern of fluorescence signals among different effectors. (A) Cos-1 cells expressing VN-Ras G12V and RBDs-VC as indicated were observed with confocal microscopy, and $x y, x z$, and $y z$ images are shown. The right panel is a higher magnification image of the inset. (B) Cos-1 cells were transfected with expression vectors for pCAGGS-VNRas G12V and RBDs-VC. After $24 \mathrm{~h}$, the cells were fixed with $4 \%$ paraformaldehyde, and stained with anti-EEA1 monoclonal antibody, followed by further incubation with Alexa 594conjugated anti-mouse antibody. The cells were observed with an FV-1000 confocal microscope. The lowest panels are higher magnification photographs of the merged image insets. (C) The cells expressing VN-Ras G12V and RBDs-VC were imaged as in (B), and the numbers of vesicles were quantitated. Average values of 5 cells are shown with S.D. ${ }^{*}, P<0.01$.

Fig. 3. The BiFC signal emanated by Ras-PI3K is associated with the endocytotic pathway. (A) Cos-1 cells were transfected with expression vectors for pCAGGS-VN-Ras WT and PI3K-RBDVC. After $24 \mathrm{~h}$, the cells were stimulated by EGF, fixed with paraformaldehyde, stained with EEA1, and then observed with confocal microscopy. (B) Cos-1 cells expressing VN-Ras WT and 
RBDs-VC were analyzed as in (A). The number of vesicles configured by BiFC and EEA1 was quantitated, and the ratios of colocalization/EEA1 before and after EGF stimulation were plotted. *, $P<0.05$; NS, not significant. (C, D) Cos-1 cells were transfected with expression vectors for VN- Ras-WT and PI3K-RBD-VC. After $24 \mathrm{~h}$, the cells were serum-starved and stimulated by $100 \mathrm{ng} / \mathrm{ml}$ EGF. (C) Selected fluorescence images at the indicated time (min) are shown. (D) Kymographic analysis of the vesicular movement. Images of the regions containing vesicles taken over $20 \mathrm{~min}$ are displayed. The top and bottom of the images represent periphery (p) and center (c) of the cells, respectively, and arrow heads indicate vesicles. Bar, 1 min. (E, F) Cos-1 cells expressing VN-Ras and PI3K-RBD-VC were serum-starved, incubated at $16^{\circ} \mathrm{C}$ for $1 \mathrm{~h}$ or left untreated, and then stimulated by EGF. (E) Representative images are shown. (F) Number of vesicles configured by BiFC was quantitated and average values are shown with standard deviations. *, $P<0.05$; NS, not significant.

Fig. 4. PI $3 \mathrm{~K}$ recruitment to the endosome by Ras. (A) Cells expressing Myc-PI3K p110 $\gamma$ alone and cells expressing Myc-PI3K p110 $\gamma$ with Ras WT or Ras G12V were stained with anti-Myc antibody and imaged using confocal microscopy. The lowest panels are higher magnification photographs of the insets. (B) Cos-1 cells expressing HA-PI3K and GFP-Ras G12V were stained with anti-HA rat monoclonal and anti-EEA1 mouse monoclonal antibodies, incubated with AlexaFluor647-conjugated anti-rat and AlexaFluor594-conjugated anti-mouse antibodies, and then imaged on a confocal microscope. (C) Fluorescence intensities of Alexa594 (blue), GFP (green), and Alexa647 (red) along with the line in the merged image in (B) were plotted from a to $b$. Note that overlapping peaks indicate colocalization.

Fig. 5. PI3K recruitment by Ras regulates $\mathrm{PIP}_{3}$ production in the endosome. (A, B) Rasdependent $\mathrm{PIP}_{3}$ accumulation in the endosome. Cos-1 cells expressing AKT-PH-CFP with or without a dominant negative mutant of H-Ras (H-Ras S17N) were stimulated by EGF (A). Fluorescence intensity at the plasma membrane was monitored, and average fold increase is shown with S.D. *, $P<0.05$; NS, not significant (B). (C) The cells were prepared as in (A) and 
Tsutsumi, K. et al.

were subjected to immunofluorescence to visualize EEA-1. (D) Vesicles formed by Akt-PH and EEA1 were counted. Average values of 5 cells are shown with S.D. (E) Triplecolor-imaging for simultaneous visualization of $\mathrm{PIP}_{3}$ production, $\mathrm{BiFC}$, and endosomes. Cos- 1 cells expressing VN-Ras, PI3K-RBD-VC, and Akt-PH-CFP were processed as in (C). The panel at the lower right is a higher magnification photograph of the inset. (F) Fluorescence intensities of CFP (blue), YFP (green), and Alexa594 (red) along with the line in the merged image in (E) were plotted from a to b. (G) Cos-1 cells were co-transfected with an expression vector for Akt-PH-CFP and those for the effector-domain mutants of H-Ras G12V as indicated. The number of vesicles formed by Akt-PH were quantitated and plotted. *, $P<0.002$. Of note, Y40C, D38E, and E37G specifically bind to PI3K, Raf, and RalGDS, respectively.

\section{Legend for Supplementary Movies}

Supplementary Movie S1 Quick Time Movie associated with Fig. 3c 


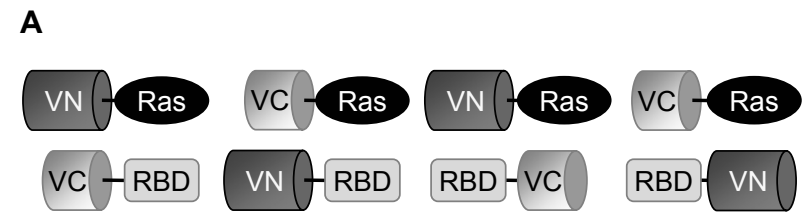

B

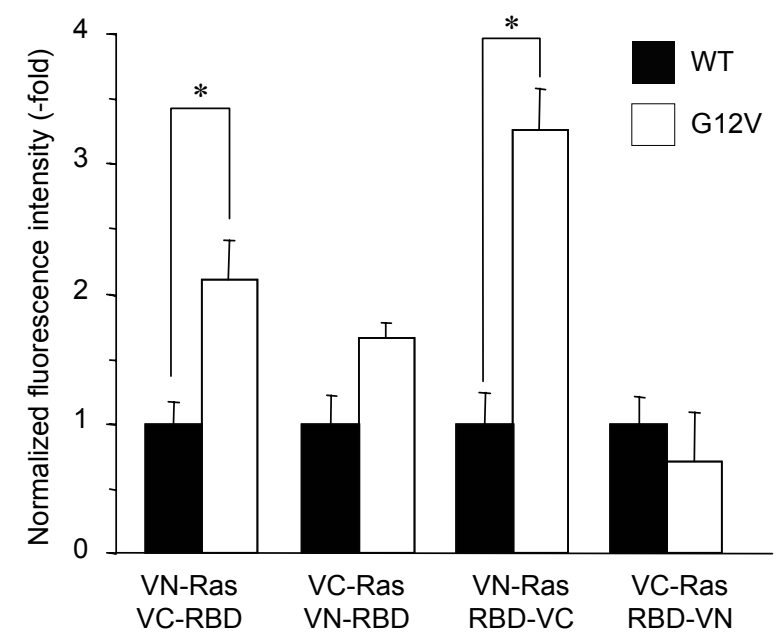

Figure 1. Tsutsumi, K. et al. 


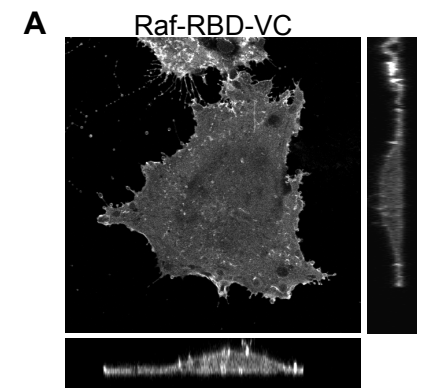

B
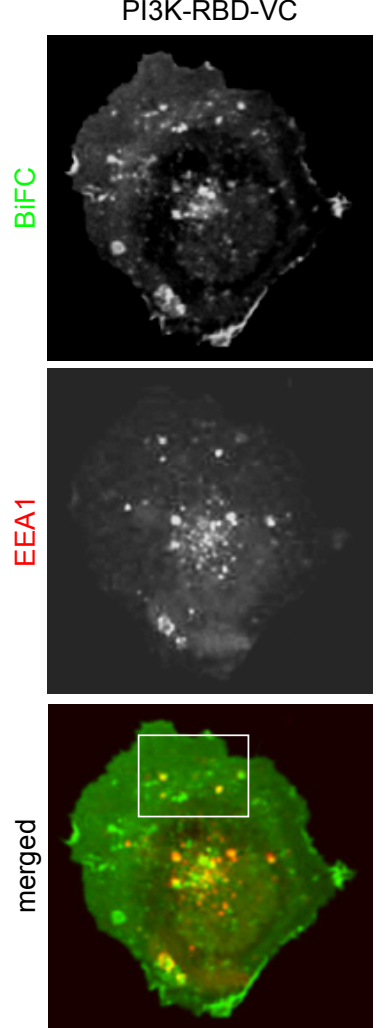

$\stackrel{\varpi}{\Phi}$

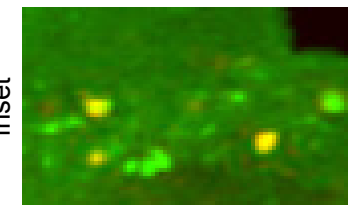

RalGDS-RBD-VC

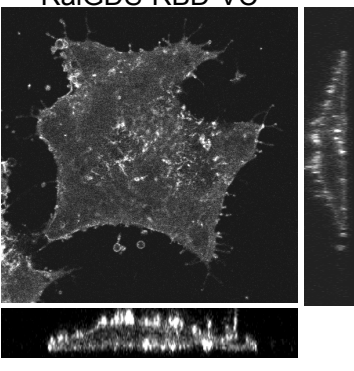

Raf-RBD-VC
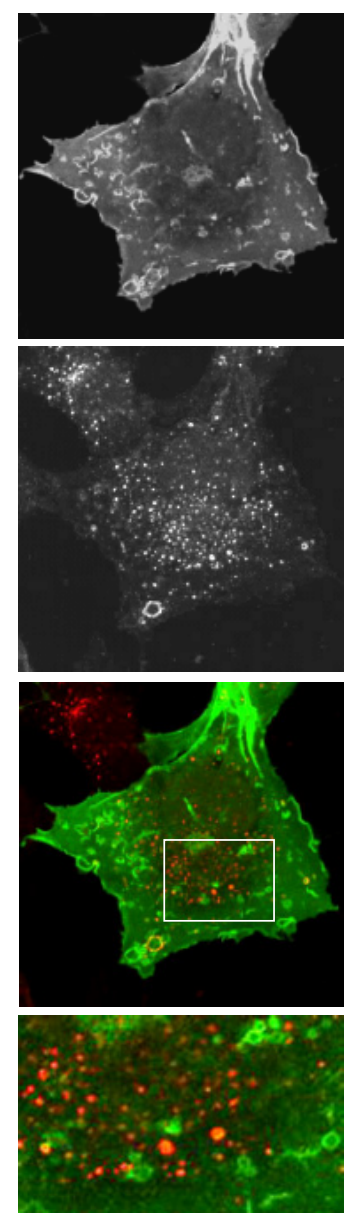

PI3K-RBD-VC

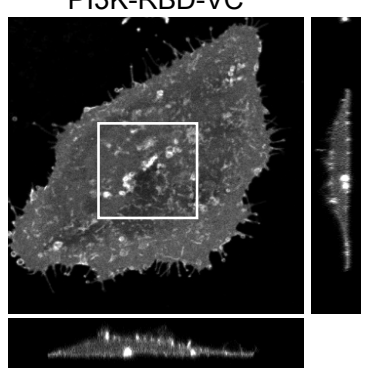

RalGDS-RBD-VC
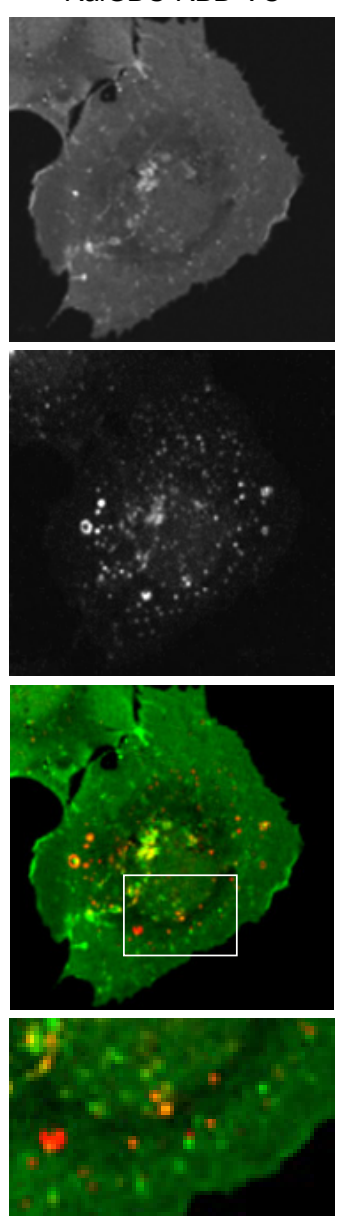

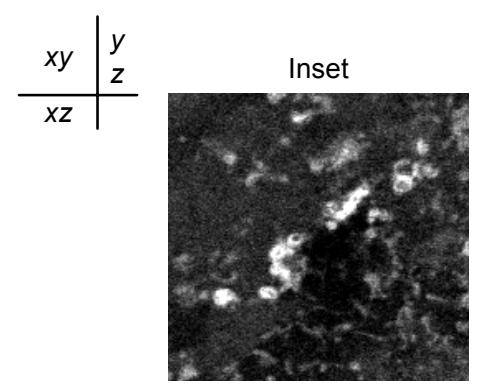

C

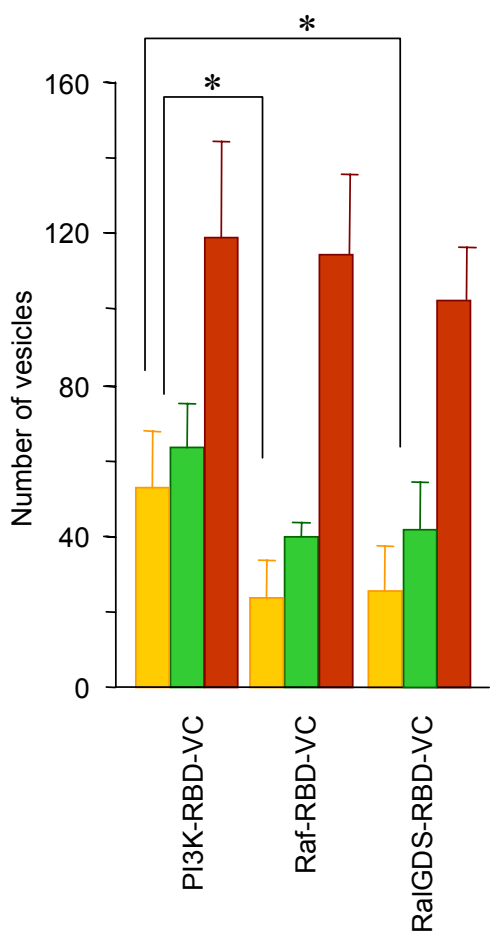

BiFC (+); EEA1 (+)

$\square \operatorname{BiFC}(+)$

EEA1 (+)

Figure 2. Tsutsumi, K. et al. 


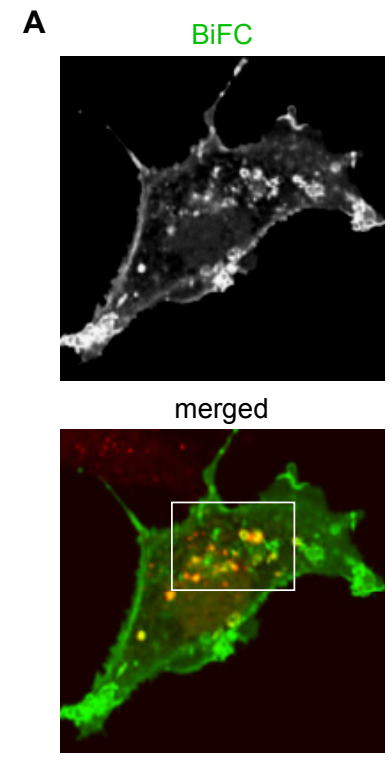

B

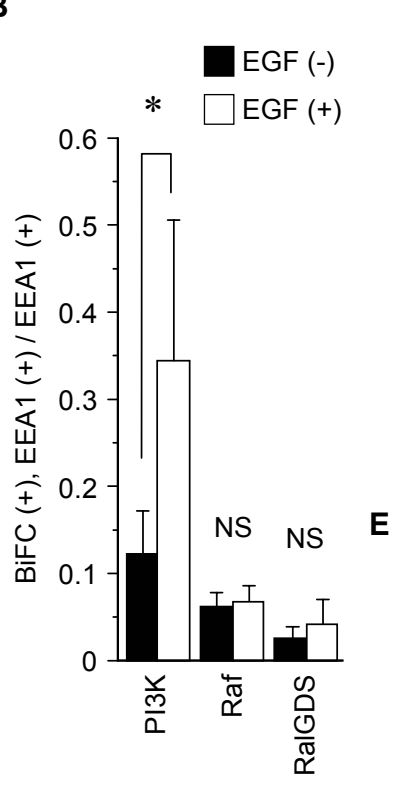

D
C
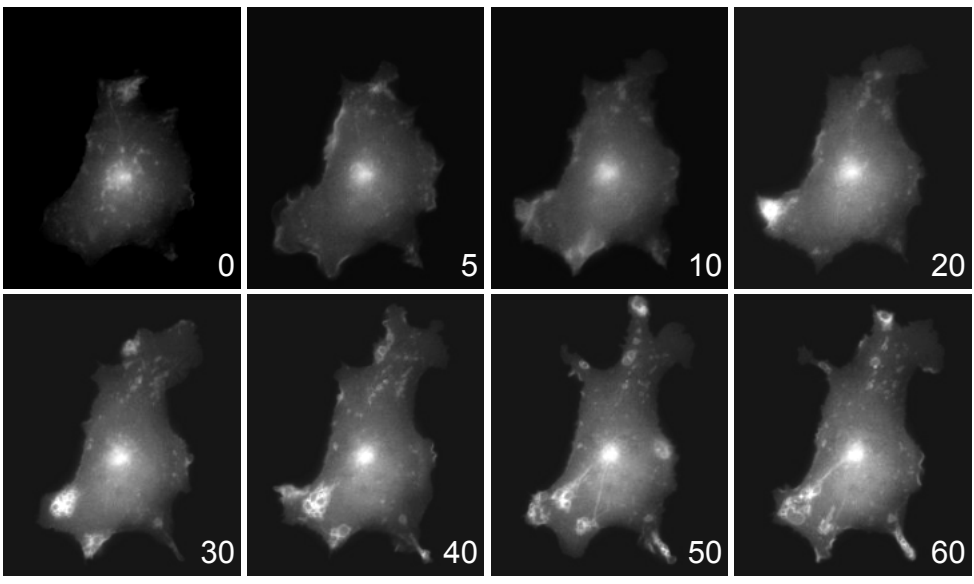
30

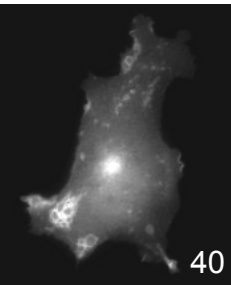

$F$
$-1 \mathrm{~min}$
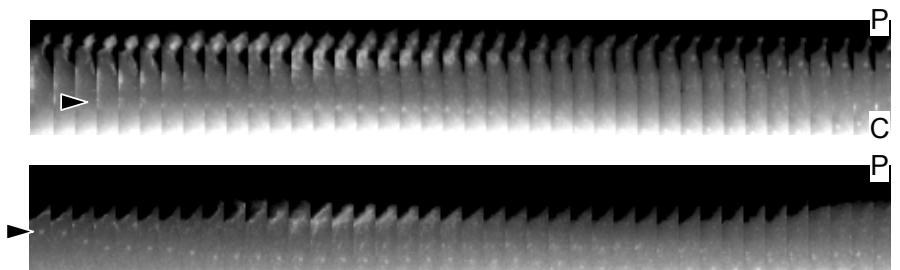

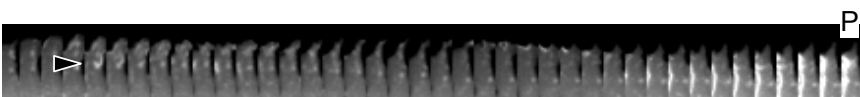

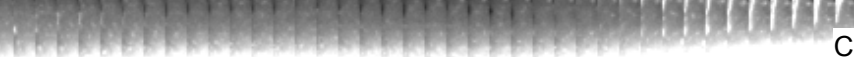
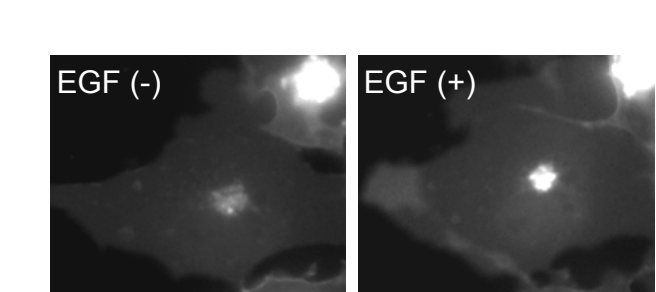

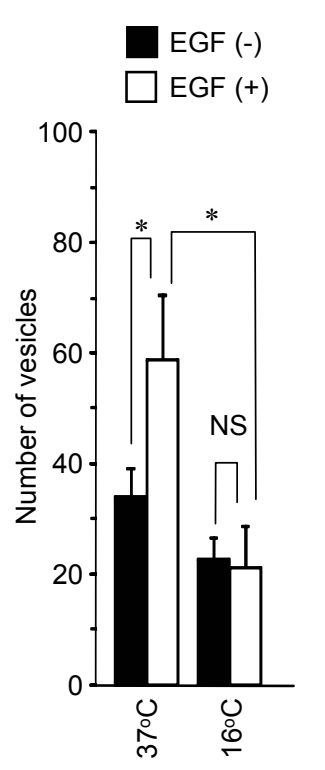

Figure 3 .Tsutsumi, K. et al. 


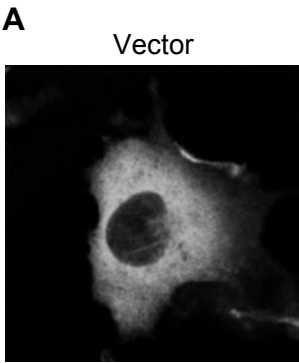

Ras WT + EGF

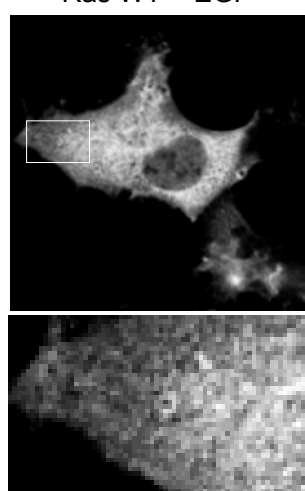

Ras WT

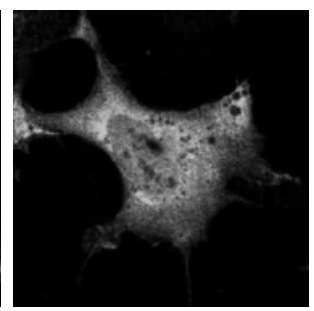

Ras G12V

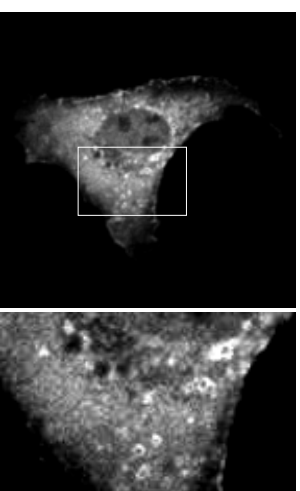

B GFP (Ras G12V)

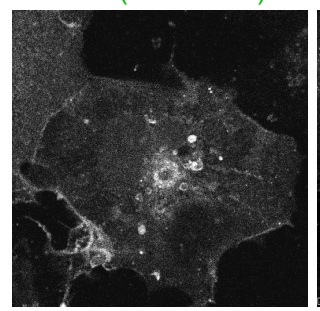

Alexa647 (PI3K)
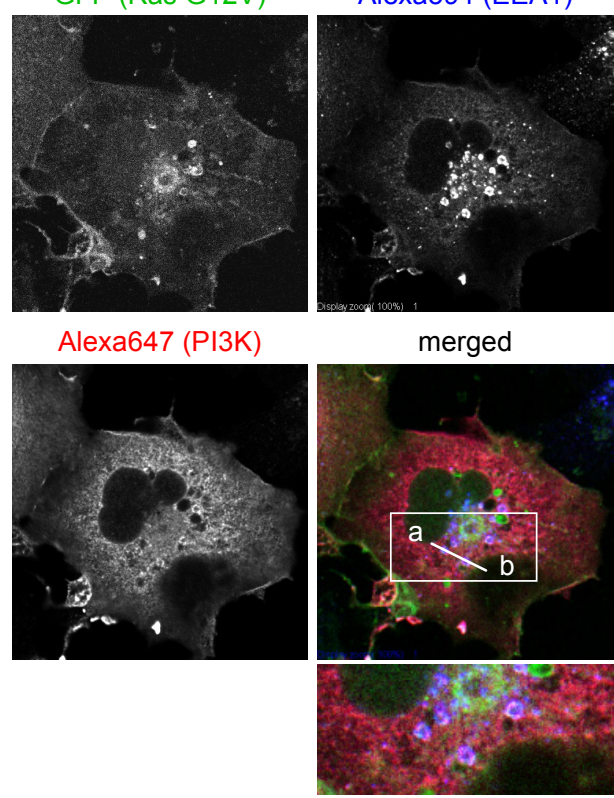

merged
C

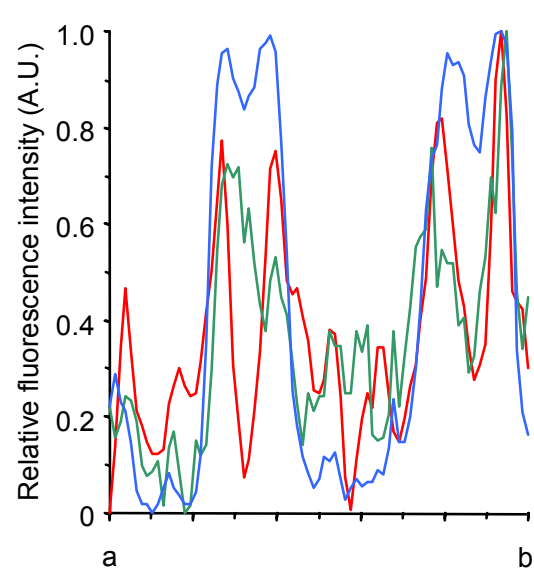

Figure 4. Tsutsumi, K. et al. 

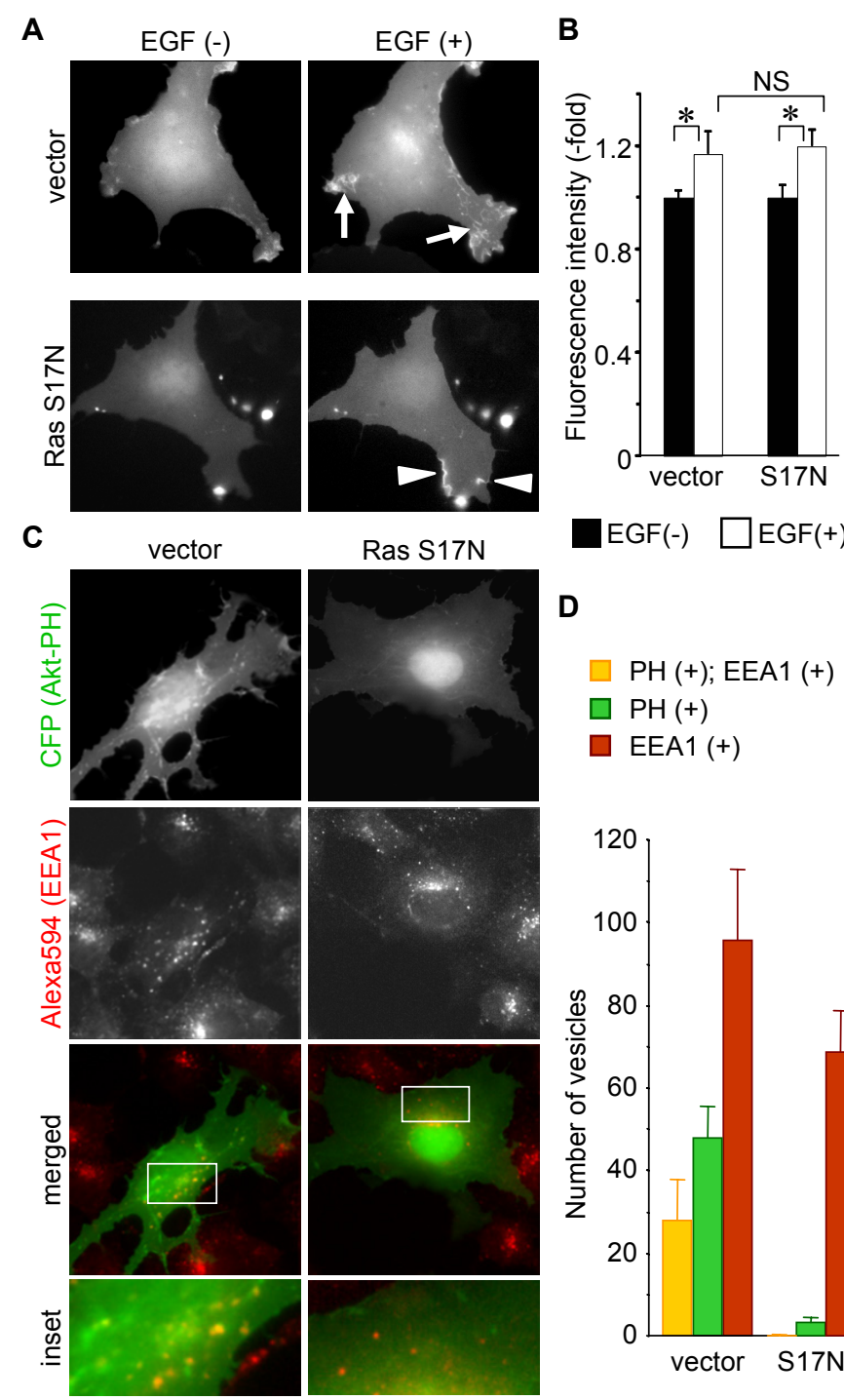

E
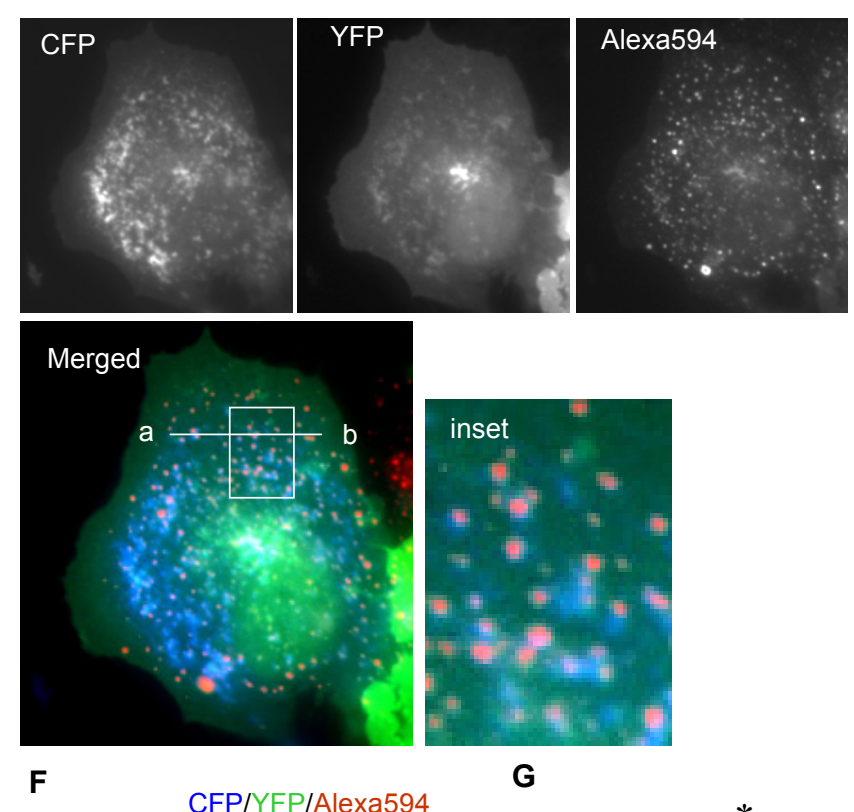

EGF(-) $\square$ EGF(+)

D

$\square \mathrm{PH}(+)$; EEA1 (+)

$\square \mathrm{PH}(+)$

$\square \operatorname{EEA1}(+)$
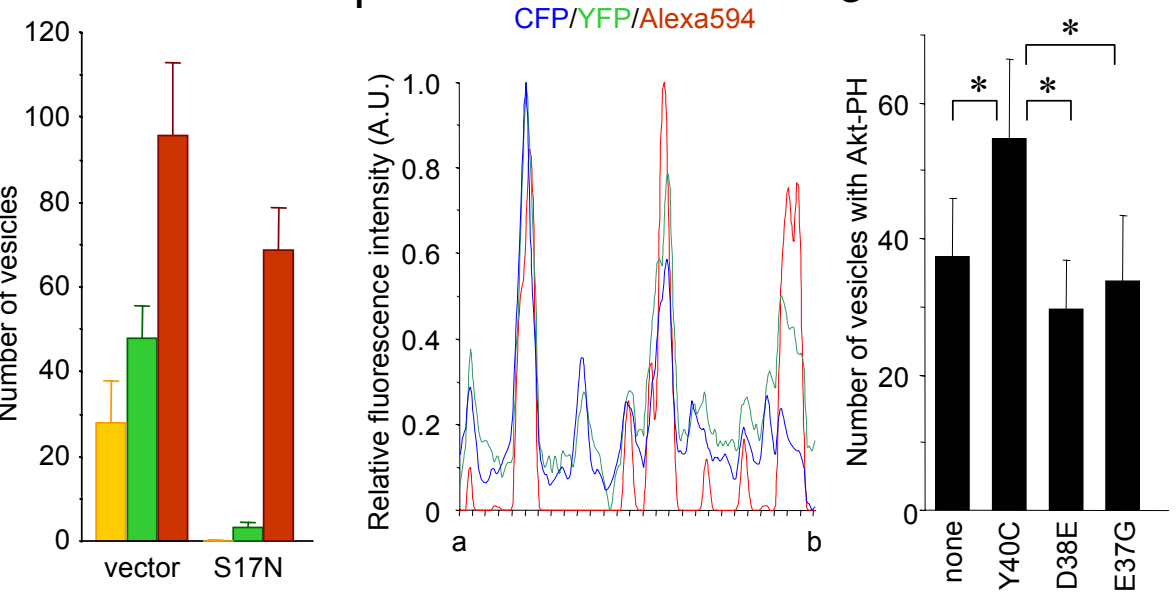

Figure 5. Tsutsumi, K. et al. 